\title{
Comparação do Perfil Epidemiológico, Clínico e dos Resultados das Intervenções Coronárias Percutâneas entre os Gêneros Masculino e Feminino, na População Brasileira: Dados do Registro CENIC
}

Marcelo Antônio Cartaxo Queiroga Lopes ${ }^{1}$, Marco Antonio De Vivo Barros ${ }^{1}$, Itamar Ribeiro de Oliveira ${ }^{1}$, Helman Campos Martins², Maria Sanali Paiva², João Alfredo Cunha Lima'1, Gustavo Souto Maior², Hugo Diógenes de Oliveira Paiva ${ }^{3}$, Luiz Alberto Mattos ${ }^{4}$, José Antonio Marin-Neto ${ }^{5}$,

pelos colaboradores da CENIC*

\section{RESUMO}

Fundamentos: No cenário dos estudos controlados e randomizados, em mulheres, a doença arterial coronária (DAC) apresenta maiores desafios no que se refere ao diagnóstico e ao tratamento. Essas noções são mais controversas no âmbito do chamado "mundo real", especialmente no que tange ao tratamento por intervenção coronária percutânea (ICP). Objetivo: Comparar os resultados de procedimentos de ICP, na fase hospitalar, em brasileiras e brasileiros. Método: Análise retrospectivamente idealizada a partir dos dados inseridos no registro da Central Nacional de Intervenções Cardiovasculares (CENIC), em sua presente forma de captura eletrônica, conforme ficha padronizada, desde o início de 1999 até o final de 2007: características demográficas e da história clínica, inclusive fatores de risco no contexto da DAC; detalhes técnicos dos procedimentos, de seu sucesso e complicações; tratamento farmacológico adjunto. Resultados: No período, 197.139 intervenções coronárias foram registradas, sendo 131.797 (66,85\%) em homens e 65.342 (33,15\%) em mulheres. A média de idade foi significativamente superior nas mulheres $(64,0 \pm 11,6$ anos vs. $60,4 \pm 11,7$ anos; $\mathrm{p}<0,0001)$, que apresentaram maior prevalência de diabetes melito $(39,4 \%$ vs. $28,5 \%$; p < 0,0001). Nos homens, houve maior prevalência de hipertensão arterial $(25,6 \%$ vs. $28,9 \%$; p < 0,0001), tabagismo $(19,1 \%$ vs. $34,0 \% ; p<0,0001)$, antecedentes de infarto do miocárdio

\footnotetext{
Cardiocenter - Hospital Santa Paula - João Pessoa, PB.

InCor - Natal, RN.

Natal Hospital Center - Natal, RN.

4 Instituto Dante Pazzanese de Cardiologia - São Paulo, SP.

5 Divisão de Cardiologia, Hospital das Clínicas da Faculdade de Medicina de Ribeirão Preto, USP - Ribeirão Preto, SP.

Correspondência: Marcelo Antônio Cartaxo Queiroga Lopes. Av.

Max Zagel, 91 - Cabedelo, PB - CEP 58310-000

E-mail: mqueiroga@terra.com.br

Recebido em: 5/9/2008 • Aceito em: 9/12/2008
}

\section{SUMMARY}

Comparison of the Epidemiologic and Clinical Profiles and Results of Percutaneous Coronary Interventions in Men and Women in Brazil: The CENIC Registry Data

Background: There are major challenges in diagnosing and treating women with coronary artery disease (CAD), particularly in randomized and controlled studies. These notions are more controversial in the real world, especially with respect to percutaneous coronary intervention $(\mathrm{PCI})$. Objective: To compare results from in-hospital $\mathrm{PCl}$ procedures in male and female Brazilian patients. Method: A retrospective analysis was done, based on data electronically uploaded into the CENIC (Central Nacional de Intervenções Cardiovasculares) Registry. A standardized form to gather data from patients treated between the beginning of 1999 to the end of 2007 was used and included clinical, angiographic and procedural results, including clinical success and complications. Results: In this period, 197,139 coronary interventions were reported; $131,797(66.85 \%)$ in men and $65,342(33.15 \%)$ in women. Women were older $(64.0 \pm 11.6$ yrs vs. $60.4 \pm 11.7$ yrs; $\mathrm{p}<0.0001)$, and had a higher prevalence of diabetes mellitus $(39.4 \%$ vs. $28.5 \%$; $p<0.0001)$. In contrast, men showed a higher prevalence of hypertension $(25.6 \%$ vs. $28.9 \% ; p<0.0001)$, smoking (19.1\% vs. $34.0 \% ; p<0.0001)$,

* Lista de colaboradores em Apêndice 
Lopes MACQ, et al. Comparação do Perfil Epidemiológico, Clínico e dos Resultados das Intervenções Coronárias Percutâneas entre os Gêneros Masculino e Feminino, na População Brasileira: Dados do Registro CENIC. Rev Bras Cardiol Invas. 2008;16(4):463-473.

$(21,1 \%$ vs. $26,8 \% ;$ p $<0,0001)$, de ICP $(17,5 \%$ vs. $19,3 \%$; $p<0,0001)$ e de revascularização miocárdica cirúrgica prévia $(8,3 \%$ vs. $11,0 \% ; p<0,0001)$. Em pacientes com ICP prévia, a nova intervenção ocorreu mais freqüentemente nos homens por progressão da doença, novas lesões $(56,6 \%$ vs. $59,5 \% ; p<0,006)$, e, nas mulheres, por reestenose $(39,2 \%$ vs. $36,4 \% ; p<0,0001)$. A ICP foi realizada nas mulheres em maior número de vezes que nos homens por indicações clínicas instáveis $(56,5 \%$ vs. 55,8\%; $p=0,003)$. Angiograficamente, verificou-se proporção maior de lesões de um único vaso entre as mulheres $(53,2 \%$ vs. 49,3\%; p < 0,0001), e menor prevalência de disfunção ventricular esquerda grave $(4,1 \%$ vs. $4,6 \%$; $p<0,0001)$, de lesões complexas (B2 e C) $(64,3 \%$ vs. $66,0 \% ; p<0,0001)$, de trombo visível $(14,6 \%$ vs. $17,6 \%$; $\mathrm{p}<0,0001$ ), de lesões extensas (superiores a $20 \mathrm{~mm}$ ) $(26,6 \%$ vs. $28,1 \%$; p < 0,0001) e de envolvimento de ramos secundários $(26,6 \%$ vs. 27,6\%; p < 0,0001). Em contraposição, nas mulheres observou-se mais calcificação $(23,4 \%$ vs. $22,8 \%$; $p<0,0001)$, e menor diâmetro $(2,95 \mathrm{~mm} \pm$ $0,66 \mathrm{~mm}$ vs. $3,04 \mathrm{~mm} \pm 0,75 \mathrm{~mm} ; \mathrm{p}=0,001)$ e extensão $(17,2 \mathrm{~mm} \pm 7,1 \mathrm{~mm}$ vs. $17,7 \mathrm{~mm} \pm 7,3 \mathrm{~mm} ; \mathrm{p}=0,04)$ dos stents implantados. As mulheres tenderam a ter taxa mais elevada de insucesso $(0,75 \%$ vs. $0,68 \% ; p=0,077)$, e efetivamente apresentaram maiores taxas de óbito $(1,20 \%$ vs. $0,79 \% ; p<0,0001)$ e de infartos miocárdicos nãofatais $(0,54 \%$ vs. $0,41 \%$; $p<0,0001)$. Conclusões: A ICP em mulheres brasileiras apresenta resultados imediatos discretamente menos satisfatórios que nos homens, em associação à tríade de idade mais avançada, maior prevalência de diabetes melito e menor calibre do vaso.

DESCRITORES: Angioplastia transluminal percutânea coronária. Angioplastia. Gênero e saúde. Fatores sexuais. Fatores de risco. Resultado de tratamento. previous myocardial infarction $(21.1 \%$ vs. $26.8 \%$; $p<0.0001)$, previous $\mathrm{PCl}(17.5 \%$ vs. $19.3 \% ; \mathrm{p}<0,0001)$ and previous coronary artery bypass surgery $(8.3 \%$ vs. $11.0 \% ; p<0.0001)$. In male patients with previous $\mathrm{PCl}$, new interventions were done more frequently due to disease progression $(56.6 \%$ vs. $59.5 \% ; p<0.006)$ whereas in women they were done predominantly due to restenosis $(39.2 \%$ vs. $36.4 \% ; \mathrm{p}<0.0001)$. $\mathrm{PCl}$ was performed in women more often than in men due to unstable clinical conditions $(56.5 \%$ vs. $55.8 \%$; $p=0.003)$. Angiographically, a higher number of single vessel lesions was noted among women $(53.2 \%$ vs. $49.3 \% ; p<0.0001)$ as well as a lesser prevalence of severe left ventricle dysfunction $(4.1 \%$ vs. $4.6 \% ; \mathrm{p}<0.0001)$, complex lesions (B2 and C) $(64.3 \%$ vs. $66.0 \% ; p<0.0001)$, visible thrombus $(14.6 \%$ vs. $17.6 \%$; $\mathrm{p}<0.0001)$, lesions $>20 \mathrm{~mm}(26.6 \%$ vs. $28.1 \%$; $p<0.0001)$ and involvement of secondary branches $(26.6 \%$ vs. $27.6 \%$; $\mathrm{p}<0.0001)$. In contrast, in women there was higher rate of calcified lesions $(23.4 \%$ vs. $22.8 \%$; $p<0.0001)$ and a smaller vessel diameter $(2.95 \mathrm{~mm} \pm 0.66 \mathrm{~mm}$ vs. $3.04 \mathrm{~mm}$ $\pm 0.75 \mathrm{~mm} ; \mathrm{p}=0,001)$ and stent length $(17.2 \mathrm{~mm} \pm 7.1 \mathrm{~mm}$ vs. $17.7 \mathrm{~mm} \pm 7.3 \mathrm{~mm} ; \mathrm{p}=0.04$ ) was observed. Women tend to have a higher rate of unsuccessful procedures $(0.75 \%$ vs. $0.68 \% ; p=0,077)$, and presented higher rates of death $(1.20 \%$ vs. $0.79 \% ; p<0.0001)$ and non-fatal myocardial infarction $(0.54 \%$ vs. $0.41 \%$; $p<0.0001)$. Conclusion: $\mathrm{PCl}$ performed in female Brazilian patients showed a slightly less favorable in-hospital results compared to male patients and was associated, among other factors, to a triad of more advanced age, higher prevalence of diabetes mellitus and, probably, a smaller vessel diameter.

DESCRIPTORS: Angioplasty, transluminal, percutaneous coronary. Angioplasty. Gender and health. Sex factors. Risk factors. Treatment outcome.
C omparativamente aos homens, no contexto da doença arterial coronária (DAC), as mulheres apresentam múltiplos aspectos desconcertantes'. Por exemplo, entre outros fatores, os hormônios naturais as protegem nitidamente quanto à prevalência de DAC, enquanto permanecem em idade fértil ${ }^{2}$. Entretanto, a terapia de reposição hormonal não se mostrou eficaz, após a menopausa, seja como prevenção secundária seja como medida preventiva primária ${ }^{3,4}$. Isso, a despeito dos indícios de proteção que advinham de estudos observacionais anteriores, que, aparentemente, corroboravam a atraente hipótese fisiopatológica então prefigurada ${ }^{5}$. As mulheres apresentam maiores problemas para o diagnóstico da DAC estável, são encaminhadas para angiografia menos freqüentemente, e recebem menos tratamento clínico e de revascularização miocárdica que homens ${ }^{6}$. Por conta desses fatores, e provavelmente de outros ainda não esclarecidos, como verdadeira nêmese fisiopatológica, oferecem maiores riscos prognósticos diante de qualquer das modalidades terapêuticas empregadas, na forma crônica da DAC, e no contexto do infarto agudo do miocárdio com supradesnivelamento de ST, ainda que não necessariamente nas outras síndromes isquêmicas miocárdicas instáveis $(\mathrm{SIMI})^{7}$.

Mesmo após a consolidação da intervenção coronária percutânea (ICP), em sua forma de atuação com stents, passou-se a relatar, também nesse contexto, o prognóstico adverso das mulheres relativamente à população masculina, em qualquer das formas clínicas de DAC, em termos de resultados imediatos e também a longo prazo após o procedimento ${ }^{8-10}$.

Esse panorama mais desfavorável poderia resultar em condutas diversas para homens e mulheres, relativamente às indicações de ICP. Assim, análises de subgrupos femininos incluídos nos estudos FRISC-2 e RITA-3 permitiram questionar se a conduta intervencionista seria igualmente benéfica e aplicável às muIheres ${ }^{11,12}$. Em contraste, outras análises pré-especificadas de estudos controlados e randomizados, como o TACTICSTIMI-18 e o CADILLAC, evidenciaram que o benefício 
dos tratamentos então testados usualmente se mantinha em mulheres portando SIMI e tratadas por estratégia intervencionista ${ }^{13,14}$. Em especial para SIMI sem supradesnivelamento de ST, o benefício da estratégia intervencionista estende-se inquestionavelmente às pacientes do gênero feminino de médio e alto risco, segundo revisão metanalítica recente ${ }^{15}$.

Em síntese, o efeito gênero tem potencial de influenciar os resultados da ICP, quando aplicada nos vários cenários clínicos da DAC, e seu impacto em condições diversas daquelas relativamente artificializadas dos estudos controlados e randomizados é menos conhecido e mais controverso. No Brasil, o registro CENIC (Central Nacional de Intervenções Cardiovasculares) permite comparar o desfecho dos procedimentos de ICP realizados em dezenas de milhares de homens e mulheres, e correlacioná-lo a vários fatores e aspectos durante a fase hospitalar, capturados pelo processo de notificação. Esse foi o objetivo do presente trabalho.

\section{MÉTODO}

Este estudo consistiu de análise retrospectivamente idealizada a partir dos dados inseridos no registro CENIC, em sua presente forma de captura eletrônica, conforme ficha padronizada, desde o início de 1999 até o final de 2007, e constante no website da Sociedade Brasileira de Hemodinâmica e Cardiologia Intervencionista (www.shbci.org.br). Nesse período, as informações sobre todas as intervenções coronárias registradas na CENIC abrangeram dezenas de instituições, com participação efetiva de centenas de associados espontaneamente vinculados ao Registro, conforme lista anexa em apêndice a este manuscrito.

A inclusão das informações coletadas no Registro da CENIC foi recentemente aprovada pelo Comitê de Ética em Pesquisa do Hospital das Clínicas da Faculdade de Medicina de Ribeirão Preto da Universidade de São Paulo, instituição na qual todos os procedimentos, sem exceção, são sempre relatados à CENIC.

Os dados coletados e utilizados neste estudo são relacionados a características demográficas, da história médica, angiográficas, e dos procedimentos, incluindo as complicações da fase hospitalar. Os dados foram informados por todos os centros ou instituições envolvidas, não tendo sido realizadas análises laboratoriais centralizadas ou adjudicação de quaisquer informações clínicas, angiográficas ou detalhes dos procedimentos.

As definições de sucesso ou insucesso do procedimento, complicações da fase hospitalar (óbito, infarto do miocárdio não-fatal, etc.), são as habitualmente empregadas quando do preenchimento das fichas relativas a cada procedimento, e constam de publicações anteriores, referentes aos dados da CENIC.

As variáveis contínuas foram apresentadas como médias e desvios padrão, e as comparações foram feitas pelo testes $t$ de Student ou ANOVA, quando apropriado, para dados paramétricos, e de Wilcoxon, para dados não-paramétricos. As variáveis categóricas foram tabeladas como números absolutos e porcentagens, e comparadas com o teste do qui-quadrado. Nível de significância foi fixado para todas as comparações em $p=0,05$, sendo as análises realizadas por meio do pacote estatístico Primer of Biostatistics, versão 3.0 .

\section{RESULTADOS}

Entre $1^{\circ}$ de janeiro de 1999 e 31 de dezembro de 2007, foram cadastradas, pelo sistema eletrônico on line do Registro CENIC, 197.139 fichas correspondentes a procedimentos de intervenção coronária. Destes, $131.797(66,85 \%)$ foram executados em homens e $65.342(33,15 \%)$, em mulheres.

Na Tabela 1, aparecem as características clínicas dos dois grupos, sendo os aspectos que os diferenciaram: a média de idade, significativamente superior nas mulheres $(64,0 \pm 11,6$ anos vs. 60,4 \pm 11,7 anos; $\mathrm{p}<0,0001)$, e, também, maior prevalência, entre as mulheres, de diabetes melito $(39,4 \%$ em mulheres vs. $28,5 \%$ em homens; $p<0,0001)$. Em contraste, fatores de risco como hipertensão arterial sistêmica (25,6\% vs. $28,9 \%$; $p<0,0001)$ e tabagismo $(19,1 \%$ vs. $34,0 \%$; $p<0,0001)$ foram mais prevalentes entre os homens. Estes também tiveram mais antecedentes de infarto do miocárdio (21,1\% vs. 26,8\%; p < 0,0001), de ICP $(17,5 \%$ vs. $19,3 \%$; $p<0,0001)$ e de revascularização miocárdica cirúrgica prévias (8,3\% vs. 11,0\%; $p<0,0001)$. Em pacientes com ICP prévia, a nova intervenção ocorreu mais freqüentemente nos homens por progressão da doença, novas lesões (56,6\% vs. $59,5 \%$; $p<0,006)$, e, nas mulheres, por reestenose $(39,2 \%$ vs. $36,4 \% ; p<0,0001)$. A ICP foi realizada nas mulheres mais vezes que nos homens por causa de indicações clínicas instáveis (56,5\% vs. 55,8\%; $p=0,003)$; finalmente, a estratificação de risco pelo escore TIMI para síndromes coronárias instáveis mostrou prevalência comparável de baixa, moderada e alta gravidade entre os dois grupos.

Quanto às características angiográficas, descritas na Tabela 2, dois grupos se discriminaram com relação a: proporção maior de uniarteriais entre as mulheres (53,2\% vs. 49,3\%; p < 0,0001) e, em contraste, por maior prevalência de disfunção ventricular esquerda grave $(4,1 \%$ vs. $4,6 \% ; p<0,0001)$, de lesões complexas (B2 e C) $(64,3 \%$ vs. 66,0\%; p < 0,0001), de trombo visível $(14,6 \%$ vs. $17,6 \% ; p<0,0001)$, de lesões extensas (superiores a $20 \mathrm{~mm})(26,6 \%$ vs. 28, $1 \%$; $\mathrm{p}<0,0001)$, envolvimento de ramos secundários $(26,6 \%$ vs. 27,6\%; p $<0,0001)$ e fluxo TIMI $<3$ $(42,7 \%$ vs. 44,9\%; $p<0,0001$ entre os homens. Finalmente, o diâmetro $(2,95 \mathrm{~mm} \pm 0,66 \mathrm{~mm}$ vs. $3,04 \mathrm{~mm} \pm 0,75 \mathrm{~mm} ; \mathrm{p}=0,001)$ e a extensão $(17,2 \mathrm{~mm}$ $\pm 7,1 \mathrm{~mm}$ vs. $17,7 \mathrm{~mm} \pm 7,3 \mathrm{~mm} ; \mathrm{p}=0,04)$ dos stents 
TABELA 1

Características clínicas nos grupos de homens e mulheres

\begin{tabular}{|c|c|c|c|c|c|}
\hline \multirow[t]{2}{*}{ Variável } & \multicolumn{2}{|c|}{ Homens } & \multicolumn{2}{|c|}{ Mulheres } & \multirow[t]{2}{*}{ Valor de $\mathrm{p}$} \\
\hline & $\mathbf{n}$ & $\%$ & $\mathbf{n}$ & $\%$ & \\
\hline $\mathrm{n}$ & 131.797 & 66,86 & 65.342 & 33,14 & \\
\hline Idade (média $\pm \mathrm{DP}$ ), anos & $60,4 \pm 11,6$ & & $64,0 \pm 11,7$ & & $<0,001$ \\
\hline Diabetes melito & 24.131 & 28,5 & 17.723 & 39,4 & $<0,0001$ \\
\hline Hipertensão arterial & 38.089 & 28,9 & 16.728 & 25,6 & $<0,0001$ \\
\hline Tabagismo & 28.770 & 34,0 & 8.578 & 19,1 & $<0,0001$ \\
\hline Dislipidemia & 46.714 & 55,2 & 24.958 & 55,5 & 0,22 \\
\hline Antecedente familiar de ICO & 25.158 & 29,7 & 13.168 & 29,3 & 0,12 \\
\hline Infarto miocárdico prévio & 22.695 & 26,8 & 9.467 & 21,1 & $<0,0001$ \\
\hline CRM prévia & 14.461 & 11,0 & 5.425 & 8,3 & $<0,0001$ \\
\hline Intervenção coronária prévia & 25.389 & 19,3 & 11.417 & 17,47 & $<0,0001$ \\
\hline - Reestenose & 9.232 & 36,4 & 4.474 & 39,19 & 0,0001 \\
\hline - Progressão & 15.111 & 59,52 & 6.466 & 56,63 & 0,006 \\
\hline Quadro clínico & & & & & 0,003 \\
\hline - Angina estável/assintomático & 58.260 & 44,2 & 28.416 & 43,5 & \\
\hline - Síndromes coronárias agudas & 73.531 & 55,8 & 36.925 & 56,5 & \\
\hline Escore TIMI de risco (SCA) & & & & & 0,4 \\
\hline - Risco baixo & 18.815 & 40,06 & 10.199 & 39,60 & \\
\hline - Risco médio & 10.829 & 23,06 & 6.124 & 23,78 & \\
\hline - Risco elevado & 17.318 & 36,88 & 9.434 & 36,63 & \\
\hline
\end{tabular}

CRM = cirurgia de revascularização do miocárdio; DP = desvio padrão; ICO = insuficiência coronária; $\mathrm{n}$ = número de pacientes; $\mathrm{SCA}=$ síndromes coronárias agudas.

implantados foram significantemente menores nas mulheres. Houve menor uso de inibidores do complexo glicoprotéico Ilb/IIla entre as mulheres, comparativamente aos homens.

Os resultados imediatos do procedimento, expostos na Tabela 3, demonstram que os dois grupos se diferenciaram com relação a: procedimentos de urgência, mais freqüentemente realizados nos homens que nas mulheres $(13,3 \%$ vs. $12,6 \%$; $p<0,0001)$. Comparativamente aos homens, as mulheres tenderam a ter maior taxa de insucesso $(0,75 \%$ vs. $0,68 \% ; p=0,077)$, e efetivamente apresentaram mais elevadas taxas de óbito $(1,20 \%$ vs. $0,79 \% ; p<0,0001)$ e de infarto miocárdico não-fatal $(0,54 \%$ vs. $0,41 \% ; p<0,0001)$.

\section{DISCUSSÃO}

A habitualmente observada proporção de cerca de um terço apenas dos pacientes tratados com ICP ser constituído de mulheres, mais uma vez, encontrase reproduzida nos dados deste estudo ${ }^{16-20}$. Além disso, as mulheres receberam esse tratamento em fase significantemente mais avançada de suas vidas, com a média de idade em seu grupo sendo superior à dos homens em 3,6 anos. Essa diferença etária entre os dois grupos é similar à reportada em séries análogas descritas na literatura ${ }^{16-20}$.
Com relação às outras características clínicas que tradicionalmente conferem maior risco aos procedimentos de ICP, apenas a maior prevalência de diabetes melito foi registrada entre as mulheres, similarmente ao relatado na literatura ${ }^{16-20}$. Nas demais ocorreu equiparação entre os grupos ou os fatores de risco eram mais prevalentes entre os homens, como mais antecedentes de infarto agudo, de procedimentos de revascularização miocárdica, de síndromes coronarianas agudas requerendo a $\mathrm{ICP}$, de mais elevado risco clínico nesse contexto (escore TIMI), e de mais disfunção grave do ventrículo esquerdo.

Também, quanto às características angiográficas usualmente indigitando pior prognóstico, verificou-se nítido predomínio entre os homens de: lesões mais complexas, extensas (inclusive requerendo implante de stents mais longos), e em múltiplos vasos, envolvendo ramificações, com mais trombos detectados angiograficamente, mais intensa redução do grau TIMI de fluxo, e correspondente maior percepção visual de circulação colateral.

Entretanto, nesse contexto angiográfico, deve-se assinalar o significativamente menor diâmetro dos stents implantados nas mulheres, característica essa que deve expressar indiretamente também o mais reduzido calibre coronário de referência no grupo feminino. Esse 
TABELA 2

Características angiográficas dos pacientes e procedimentos

\begin{tabular}{|c|c|c|c|c|c|}
\hline \multirow[t]{2}{*}{ Variável } & \multicolumn{2}{|c|}{ Homens } & \multicolumn{2}{|c|}{ Mulheres } & \multirow[t]{2}{*}{ Valor de $p$} \\
\hline & $\mathbf{n}$ & $\%$ & $\mathbf{n}$ & $\%$ & \\
\hline Número de vasos acometidos & & & & & $<0,0001$ \\
\hline 1 & 64.996 & 49,32 & 34.763 & 53,20 & \\
\hline 2 & 42.031 & 31,89 & 19.932 & 30,51 & \\
\hline 3 & 24.293 & 18,43 & 10.360 & 15,86 & \\
\hline Disfunção sistólica (VE) grave & 6.317 & 4,57 & 2.692 & 4,12 & $<0,0001$ \\
\hline Tipo de lesão (AHA/ACC) & & & & & 0,209 \\
\hline A & 7.722 & & 4.205 & & \\
\hline B1 & 49.357 & & 25.028 & & \\
\hline B2 & 73.898 & & 35.788 & & \\
\hline C & 36.985 & & 16.925 & & \\
\hline Lesão B2+C (AHA/ACC) & 110883 & 66,02 & 52713 & 64,33 & $<0,0001$ \\
\hline Calcificação & 38.341 & 22,83 & 58.425 & 23,38 & $<0,0001$ \\
\hline Trombo visível & 29.502 & 17,56 & 11.967 & 14,60 & $<0,0001$ \\
\hline Lesão > 20 mm & 47.218 & 28,11 & 21.826 & 26,63 & $<0,0001$ \\
\hline Ramos secundários envolvidos & 46.369 & 27,61 & 21.777 & 26,57 & $<0,0001$ \\
\hline Fluxo TIMI < 3 pré-ICP & 59,123 & 44,86 & 27,925 & 42,74 & $<0,0001$ \\
\hline 0 & 27.069 & & 12.216 & & \\
\hline 1 & 9.977 & & 4.968 & & \\
\hline 2 & 22.077 & & 10.741 & & \\
\hline Circulação colateral para o vaso tratado & 13.485 & 10,23 & 6,082 & 9,31 & $<0,0001$ \\
\hline ICP com balão & 24.190 & 14,40 & 12.093 & 14,76 & 0,02 \\
\hline ICP com balão + stent & 143.772 & 85,60 & 69.853 & 85,24 & 0,86 \\
\hline Uso de abciximab adjunto à ICP & 2.915 & 2,54 & 1.347 & 2,39 & 0,04 \\
\hline Uso de tirofiban adjunto à ICP & 4.800 & 4,19 & 2.163 & 3,84 & 0,03 \\
\hline Diâmetro do stent (média \pm DP), mm & $3,04 \pm 0,75$ & & $2,95 \pm 0,66$ & & 0,001 \\
\hline Comprimento do stent (média \pm DP), mm & $17,7 \pm 7,3$ & & $17,2 \pm 7,1$ & & 0,04 \\
\hline Número de stents/paciente & $1,19 \pm 0,3$ & & $1,17 \pm 0,2$ & & 0,30 \\
\hline
\end{tabular}

$\mathrm{ACC}=$ American College of Cardiology; $\mathrm{AHA}=$ American Heart Association; $\mathrm{DP}=$ desvio padrão; ICP = intervenção coronária percutânea; $\mathrm{n}=$ número de pacientes; $\mathrm{VE}=$ ventrículo esquerdo.

aspecto tem sido relativamente negligenciado em relatos comparando homens e mulheres tratados com ICP. Nos artigos em que o diâmetro de referência do vaso tratado foi diretamente mencionado, registrou-se calibre vascular estatisticamente menor em mulheres ${ }^{16,17}$. Além disso, em revisões sobre o tema, o menor calibre coronário em mulheres é apontado como fator preponderante em tornar os procedimentos mais dificultosos até o ponto de comprometer os resultados imediatos e o prognóstico a longo prazo ${ }^{21,22}$.

Nesse cenário clínico e angiográfico em que poucas características prognosticamente negativas eram predominantes entre as mulheres, em comparação com os homens, de forma surpreendente os resultados imediatos dos procedimentos foram significantemente piores no grupo feminino. Essa observação foi registrada não somente quanto a desfechos essencialmente meca- nísticos e básicos dos procedimentos, como nítida tendência a insucesso $(p=0,077)$ e significativamente maior necessidade de revascularização emergencial, porém, e ainda mais marcantemente, de desfechos clinicamente relevantes, como mais elevadas taxas de mortalidade e de infarto agudo do miocárdio no período hospitalar.

Assim, pode-se constatar que, no cenário brasileiro, a angioplastia coronária realizada em mulheres, do ponto de vista de resultados imediatos, incluídos os desfechos clinicamente relevantes e o próprio sucesso dos procedimentos, se torne mais ominosa pela particular combinação de idade mais avançada, menor calibre luminal e de mais adverso remodelamento vascular, esta última feição dependente da marcantemente maior prevalência de diabetes melito na população de gênero feminino quando recebe ICP. Essa 
Lopes MACQ, et al. Comparação do Perfil Epidemiológico, Clínico e dos Resultados das Intervenções Coronárias Percutâneas entre os Gêneros Masculino e Feminino, na População Brasileira: Dados do Registro CENIC. Rev Bras Cardiol Invas. 2008;16(4):463-473.

TABELA 3

Resultados imediatos dos procedimentos

\begin{tabular}{|c|c|c|c|c|c|}
\hline \multirow[t]{2}{*}{ Variável } & \multicolumn{2}{|c|}{ Homens } & \multicolumn{2}{|c|}{ Mulheres } & \multirow[t]{2}{*}{ Valor de $p$} \\
\hline & $\mathbf{n}$ & $\%$ & $\mathbf{n}$ & $\%$ & \\
\hline & 131.797 & & 65.342 & & \\
\hline Procedimento & & & & & $<0,0001$ \\
\hline Emergência & 15.823 & 13,30 & 7.377 & 12,59 & \\
\hline Eletivo & 103.174 & 86,70 & 51.195 & 87,41 & \\
\hline Sucesso angiográfico & 147.669 & 99,32 & 71.921 & 99,25 & NA \\
\hline Insucesso & 1.009 & 0,68 & 541 & 0,75 & 0,077 \\
\hline Não alcançou a lesão & 424 & & 199 & & \\
\hline Não ultrapassou a lesão & 405 & & 262 & & \\
\hline Não dilatou a lesão & 180 & & 80 & & \\
\hline \multicolumn{6}{|l|}{ Complicações hospitalares } \\
\hline Óbito & 1.039 & 0,79 & 783 & 1,20 & $<0,0001$ \\
\hline Infarto agudo do miocárdio & 546 & 0,41 & 352 & 0,54 & $<0,0001$ \\
\hline Cirurgia de revascularização miocárdica & 122 & 0,09 & 76 & 0,12 & 0,1 \\
\hline Eletiva & 75 & & 49 & & \\
\hline Emergência & 47 & & 27 & & \\
\hline Acidente vascular cerebral & & 0,74 & & 0,74 & 0,66 \\
\hline Hemorrágico & 15 & & 13 & & \\
\hline Isquêmico & 1.085 & & 519 & & \\
\hline Complicações vasculares graves & 1.410 & 1,07 & 738 & 1,13 & 0,2 \\
\hline Complicações vasculares leves & 2.744 & 2,08 & 1.399 & 2,14 & 0,4 \\
\hline Insuficiência renal aguda & 1.704 & 1,29 & 808 & 1,24 & 0,3 \\
\hline Oclusão do vaso & 482 & 0,37 & 246 & 0,38 & 0,7 \\
\hline Subaguda & 300 & & 148 & & \\
\hline Aguda & 182 & & 98 & & \\
\hline
\end{tabular}

tríade confere pior prognóstico a essa série extensa de pacientes do gênero feminino inseridas no Registro CENIC, contrabalançando inclusive o peso dos outros fatores de risco clínico e angiográfico que foram preponderantes entre os homens.

Finalmente, torna-se possível concluir que os resultados da presente análise, espelhando fielmente aqueles relatados em diversos outros registros contemporâneos similares, conferem credibilidade, indiretamente, a pelo menos parte das informações processadas espontaneamente pelos associados da $\mathrm{SBHCl}$.

\section{REFERÊNCIAS BIBLIOGRÁFICAS}

1. Castelli WP. Cardiovascular disease in women. Am J Obstet Gynecol. 1988;158(6 Pt 2):1553-60.

2. Wenger NK, Speroff L, Packard B. Cardiovascular health and disease in women. N Engl J Med. 1993;329(4):247-56.

3. Hulley S, Grady D, Bush T, Furberg C, Herrington D, Riggs B, et al. Randomized trial of estrogen plus progestin for secondary prevention of coronary heart disease in postmenopausal women. Heart and Estrogen/progestin Replacement Study (HERS) Research Group. JAMA. 1998; 280(7):605-13.

4. Rossouw JE, Anderson GL, Prentice RL, LaCroix AZ, Kooperberg C, Stefanick ML, et al. Writing Group for the Women's Health Initiative Investigators. Risks and benefits of estrogen plus progestin in healthy postmenopausal women: principal results From the Women's Health Initiative randomized controlled trial. JAMA. 2002;288(3):321-33.

5. Nelson HD, Humphrey LL, Nygren P, Teutsch SM, Allan JD. Postmenopausal hormone replacement therapy: scientific review. JAMA. 2002;288(7):872-81.

6. Daly C, Clemens F, Lopez Sendon JL, Tavazzi L, Boersma E, Danchin N, et al. Euro Heart Survey Investigators. Gender differences in the management and clinical outcome of stable angina. Circulation. 2006;113(4):490-8.

7. Bradshaw PJ, Thompson PL. Sex in the CCU: women with non-ST-segment elevation acute coronary syndrome may do no worse despite less intervention. Heart. 2007;93(11): 1327-8.

8. Kelsey SF, James M, Holubkov AL, Holubkov R, Cowley MJ, Detre KM. Results of percutaneous transluminal coronary angioplasty in women.1985-1986 National Heart, Lung, and Blood Institute's Coronary Angioplasty Registry. Circulation. 1993;87(3):720-7. 
Lopes MACQ, et al. Comparação do Perfil Epidemiológico, Clínico e dos Resultados das Intervenções Coronárias Percutâneas entre os Gêneros Masculino e Feminino, na População Brasileira: Dados do Registro CENIC. Rev Bras Cardiol Invas. 2008;16(4):463-473.

9. Bell MR, Grill DE, Garratt KN, Berger PB, Gersh BJ, Holmes DR Jr. Long-term outcome of women compared with men after successful coronary angioplasty. Circulation. 1995;91(12): 2876-81.

10. Malenka DJ, O'Connor GT, Quinton H, Wennberg D, Robb JF, Shubrooks S, et al. Differences in outcomes between women and men associated with percutaneous transluminal coronary angioplasty. A regional prospective study of 13,061 procedures. Northern New England Cardiovascular Disease Study Group. Circulation. 1996;94(9 Suppl):II99-104.

11. Lagerqvist B, Säfström K, Stahle E, Wallentin L, Swahn E. Is early invasive treatment of unstable coronary artery disease equally effective for both women and men? FRISC II Study Group Investigators. J Am Coll Cardiol. 2001;38(1):41-8.

12. Clayton TC, Pocock SJ, Henderson RA, Poole-Wilson PA, Shaw TR, Knight R, et al. Do men benefit more than women from an interventional strategy in patients with unstable angina or non-ST-elevation myocardial infarction? The impact of gender in the RITA 3 trial. Eur Heart J. 2004;25(18): 1641-50.

13. Glaser R, Herrmann HC, Murphy SA, Demopoulos LA, DiBattiste PM, Cannon CP, et al. Benefit of an early invasive management strategy in women with acute coronary syndromes. JAMA. 2002;288(24):3124-9.

14. Lansky AJ, Pietras C, Costa RA, Tsuchiya Y, Brodie BR, Cox $D A$, et al. Gender differences in outcomes after primary angioplasty versus primary stenting with and without abciximab for acute myocardial infarction: results of the Controlled Abciximab and Device Investigation to Lower Late Angioplasty Complications (CADILLAC) trial. Circulation. 2005;111(13): $1611-8$.
15. O'Donoghue M, Boden WE, Braunwald E, Cannon CP, Clayton TC, de Winter RJ, et al. Early invasive vs conservative treatment strategies in women and men with unstable angina and non-ST-segment elevation myocardial infarction: a meta-analysis. JAMA. 2008;300(1):71-80.

16. Mehilli J, Kastrati A, Dirschinger J, Bollwein H, Neumann FJ, Schömig A. Differences in prognostic factors and outcomes between women and men undergoing coronary artery stenting. JAMA. 2000;284(14):1799-805.

17. Jacobs AK, Johnston JM, Haviland A, Brooks MM, Kelsey SF, Holmes DR Jr, et al. Improved outcomes for women undergoing contemporary percutaneous coronary intervention: a report from the National Heart, Lung, and Blood Institute Dynamic registry. J Am Coll Cardiol. 2002;39(10):1608-14.

18. Argulian E, Patel AD, Abramson JL, Kulkarni A, Champney $\mathrm{K}$, Palmer S, et al. Gender differences in short-term cardiovascular outcomes after percutaneous coronary interventions. Am J Cardiol. 2006;98(1):48-53.

19. Berger JS, Sanborn TA, Sherman W, Brown DL. Influence of sex on in-hospital outcomes and long-term survival after contemporary percutaneous coronary intervention. Am Heart J. $2006 ; 151(5): 1026-31$.

20. Singh M, Rihal CS, Gersh BJ, Roger VL, Bell MR, Lennon RJ, et al. Mortality differences between men and women after percutaneous coronary interventions. A 25-year, single-center experience. J Am Coll Cardiol. 2008;51(24):2313-20.

21. Mikhail GW. Coronary revascularization in women. Heart. 2006;92(Suppl 3):iii19-23.

22. Benamer H, Chevalier B, Morice MC. L'angioplastie coronaire chez la femme. Ann Cardiol Angeiol (Paris). 2008; $57(6): 371-5$.

\section{APÊNDICE \\ Lista de Associados da SBHCI que se mantiveram efetivamente contribuindo com dados à CENIC, durante o biênio 2006-2007}

ABDU NEME JORGE MAKHLUF NETO
ADEMAR SANTOS FILHO
ADNAN ALI SALMAN
ADRIANO DIAS DOURADO OLIVEIRA
ADRIANO MENDES CAIXETA
ALAN NASCIMENTO PAIVA
ALAOR QUEIROZ ARAUJO FILHO
ALBERTO NAJJAR
ALCIDES FERREIRA JUNIOR
ALCIDES JOSE ZAGO
ALDO FERNANDO SOMAVILLA DUARTE
ALESSANDRA OLIVEIRA
ALESSANDRO PINA PEDROSO
ALEXANDRE DA SILVA MEDEIROS
ALEXANDRE DAMIANI AZMUS
ALEXANDRE DO CANTO ZAGO
ALEXANDRE JACKSON VON SPERLING
DE VASCONCELLOS
ALEXANDRE LOJA ANELLO
ALEXANDRE SOARES DOS SANTOS
ALEXANDRE VENTURELLI
ALEXANDRE XAVIER BRANT
ALFREDO NUNES FERREIRA FILHO

ABDU NEME JORGE MAKHLUF NETO

ADEMAR SANTOS FILHO

ADNAN ALI SALMAN

ADRIANO DIAS DOURADO OLIVEIRA

ALAOR QUEIROZ ARAUJO FILHO

ALBERTO NAJJAR

ALCIDES FERREIRA JUNIOR

ALCIDES JOSE ZAGO

A DUARTE

ALESSANDRO PINA PEDROSO

ALEXANDRE DA SILVA MEDEIROS

ALEXANDRE JACKSON VON SPERLING

DE VASCONCELLOS

ALEXANDRE LOJA ANELLO

ALEXANDRE SOARES DOS SANTOS

ALEXANDRE XAVIER BRANT

ALFREDO NUNES FERREIRA FILHO

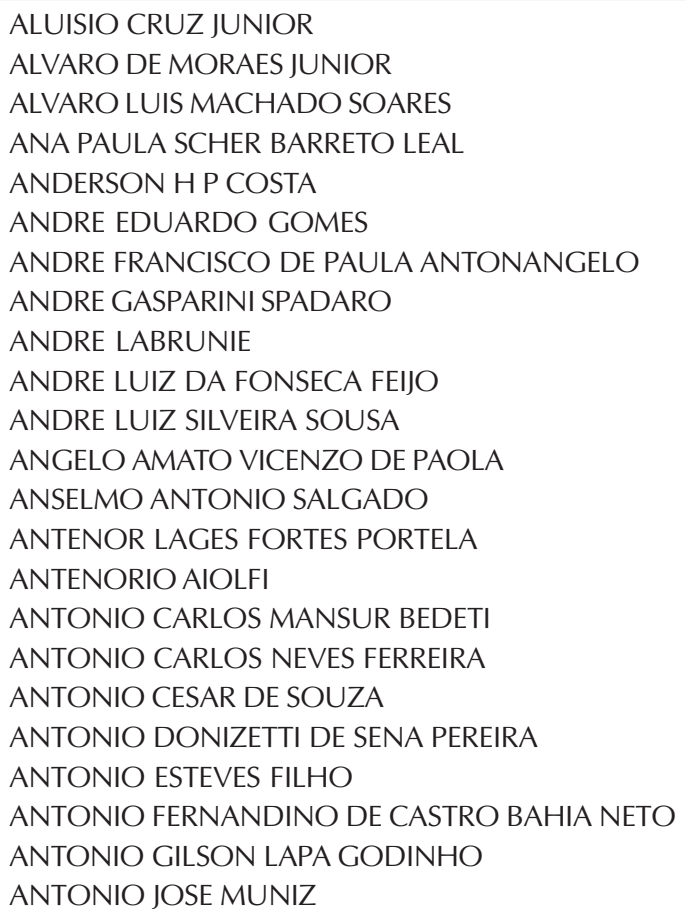


APÊNDICE (continuação)

Lista de Associados da SBHCI que se mantiveram efetivamente contribuindo com dados à CENIC, durante o biênio 2006-2007

\begin{tabular}{|c|c|}
\hline ANTONIO MORAES DE AZEVEDO JUNIOR & EDUARDO SZUSTER \\
\hline ANTONIO WATSON MENESES VIANA & ELIAS DE MELLO AYRES NETO \\
\hline ARI MANDIL & EMERSON DE ALBUQUERQUE SEIXAS \\
\hline ARTHUR LUIZ WALLBACH BARRETO & ENIO EDUARDO GUERIOS \\
\hline AUGUSTO DAIGE DA SILVA & EOLO MORANDI JUNIOR \\
\hline AUGUSTO LIMA FILHO & ERIKA PRACCHIA RIBEIRO \\
\hline BERNARDO AMORIM & ESMERALCI FERREIRA \\
\hline BRENO DE ALENCAR ARARIPE FALCAO & EULOGIO EMILIO MARTINEZ FILHO \\
\hline BRENO OLIVEIRA ALMEIDA & EUSDEMAR LIMA STEFANE \\
\hline BRUNO MOULIN MACHADO & EVANDRO GOMES DE MATOS JUNIOR \\
\hline CARLAILE ANTONIO SARMENTO DE ARAUJO COSTA & EVANDRO KARLO PRACCHIA RIBEIRO \\
\hline CARLOS AUGUSTO FORMIGA AREAS & EVANDRO LUIS QUEIROZ FLORES \\
\hline CARLOS EDUARDO DINIZ COUTO & EXPEDITO EUSTAQUIO RIBEIRO DA SILVA \\
\hline CARLOS EDUARDO FARIA SILVA & FABIANO CARAZZAI PACHECO \\
\hline CARLOS EDUARDO MAGALHAES DOMINGUES & FABIO CARDOSO DE CARVALHO \\
\hline CARLOS HENRIQUE EIRAS FALCAO & FABIO DE MEIRELES COSTA \\
\hline CARLOS ROBERTO CARDOSO & FABIO MONTEIRO MOTA \\
\hline CELMO FERREIRA DE SOUZA JUNIOR & FABIO RIDOLFI DE FIGUEIREDO \\
\hline CESAR AUGUSTO ESTEVES & FABIO SANDOLI DE BRITO JUNIOR \\
\hline CESAR ROCHA MEDEIROS & FABIO SOLANO DE FREITAS SOUZA \\
\hline CHARLES LUIZ VIEIRA & FAUSTO FERES \\
\hline CIRO JONES CARDOSO & FELIPE BORTOT CESAR \\
\hline CLACIR STAUDT & FELIPE CAMELO BIAGI \\
\hline CLARISSA CAMPO DALL ORTO & FELIPE EDUARDO HATSUMURA \\
\hline CLAUDIA MARIA RODRIGUES ALVES & FERNANDO BULLOS FILHO \\
\hline CLAUDIAMATTOS & FERNANDO DE MARTINO \\
\hline CLAUDIO AKSTEIN & FERNANDO MENDES SANT ANNA \\
\hline CLEITON DA SILVA RAMOS & FERNANDO STUCHI DEVITO \\
\hline CLEMENTE GREGUOLO & FILIPE GOLDBERG \\
\hline CONSTANTINO GONZALES SALGADO & FLAVIO ARAUJO CANEDO \\
\hline COSTANTINO ROBERTO FRACK CONSTANTINI & FLAVIO BORGES \\
\hline CRISTIANO NONATO MADUREIRA LUCENA & FLAVIO JOSE ROCHA DE SOUZA \\
\hline CYRO VARGUES RODRIGUES & FLAVIO PASSOS BARBOSA \\
\hline DANIEL FERREIRA MUGRABI & FLAVIO ROBERTO AZEVEDO DE OLIVEIRA \\
\hline DANIEL IZZET POTERIO & FRANCISCO CLAUDIO COUTO FALCAO \\
\hline DEBORAH CHRISTINA NERCOLINI & FRANCISCO JUAREZ CRUZ DE VASCONCELOS FILHO \\
\hline DECIOSALVADORIJUNIOR & FREDERICK MALTA BUARQUE DE GUSMAO \\
\hline DENIS MOULIN DOS REIS BAYERL & FREDERICO AUGUSTO LIMA E SILVA \\
\hline DIMITRI MIKAELIS ZAPPI & GALO ALFREDO MALDONADO ANDRADE \\
\hline DINALDO CAVALCANTI DE OLIVEIRA & GEORGE CESAR XIMENES MEIRELLES \\
\hline DIRCEU RODRIGUES ALMEIDA & GERALDO LUIZ DE FIGUEIREDO \\
\hline DOMENICO RODRIGO GHINELLI ZAPATER & GERSON MIRANDA \\
\hline EDISON CARVALHO SANDOVAL PEIXOTO & GIANCARLO GONCALVES \\
\hline EDMUNDO ANDRE VIVEIROS PESSANHA & GIANCARLO RABELO E SILVA \\
\hline EDMUR CARLOS DE ARAUJO & GILBERTO GUILHERME AJJAR MARCHIORI \\
\hline EDSON ADEMIR BOCCHI & GILBERTO HEINECK \\
\hline EDSON ANTONIO BREGAGNOLLO & GILBERTO LAHORGUE NUNES \\
\hline EDUARDO ARANTES NOGUEIRA & GLAUCO SOARES MAIA PIASSI \\
\hline EDUARDO FREDERICO BORSARINI FELIPE & GUILHERME ALVES \\
\hline EDUARDO HENRIQUE CURADO ELIAS & GUILHERME BRANDAO ALMEIDA \\
\hline EDUARDO JOSE PEREIRA FERREIRA & GUILHERME ESHER \\
\hline EDUARDO KEI MARQUESINI WASHIZU & GUILHERME FERRAGUTTI ATTIZZANI \\
\hline EDUARDO LUCIO NICOLELA JUNIOR & GUILHERME RODRIGUES MAFFEIS \\
\hline
\end{tabular}


APÊNDICE (continuação)

Lista de Associados da $\mathrm{SBHCl}$ que se mantiveram efetivamente contribuindo com dados à CENIC, durante o biênio 2006-2007

\begin{tabular}{|c|c|}
\hline GUSTAVOA.BARRUEO & JOSE ARY BOECHAT E SALLES \\
\hline GUSTAVO ADOLFO BRAVO RANDO & JOSE AUGUSTO MARCONDES DE SOUZA \\
\hline GUSTAVO ANDRE BARRUECO & JOSE AUGUSTO ROCHA ARAUJO \\
\hline GUSTAVO BAIOCHI VIEIRA & JOSE CARLOS FELIPE ABUD \\
\hline GUSTAVOCARVALHO & JOSE CARLOS RAIMUNDO BRITO \\
\hline GUSTAVO DE MORAES RAMALHO & JOSE DEL CARMEN SOLANO ALIAGA \\
\hline GUSTAVO ENRIQUE SANCHES ALVAREZ & JOSE ERIRTONIO FACANHA BARRETO \\
\hline GUSTAVO ITHAMAR SOUTO MAIOR & JOSE FABIO FABRIS JUNIOR \\
\hline GUSTAVO OLIVEIRA DE ALBUQUERQUE & JOSE GUILHERME CARNEIRO \\
\hline GUSTAVOSOUTO MAIOR & JOSE LUIS ARREDONDO QUINONES \\
\hline GYOVANY MENDES ALMEIDA & JOSE LUIS ATTAB DOS SANTOS \\
\hline HAROLDO CARLOS CORREA GLAVAM & JOSE MARCONI ALMEIDA SOUZA \\
\hline HEITOR GHISSONI DE CARVALHO & JOSE MARIA DIAS AZEREDO BASTOS \\
\hline HELIO JOSE CASTELLO JR & JOSE MARIANI JUNIOR \\
\hline HELIO ROQUE FIGUEIRA & JOSE MATOS BRITO CASTELLO BRANCO \\
\hline HELMAN CAMPOS MARTINS & JOSE RICARDO BUENO DE MORAES BIROLLI \\
\hline HENRIQUE ISSA ARTONI EBAID & JOSE ROBERTO MAIELLO \\
\hline HUMBERTO ALENCAR DE ARAUJO SANCHEZ & JOSE RONALDO MONT ALVERNE FILHO \\
\hline HUMBERTO MAGNO PASSOS & JOSE WALTER MENDES NOGUEIRA \\
\hline IGOR MATOS LAGO & JOSILAVIO DE ALMEIDA ARAUJO \\
\hline ITAMAR RIBEIRO DE OLIVEIRA & JULIO CESAR FRANCISCO VARDI \\
\hline IVAN FERREIRA DE FREITAS & JULIO CESAR MACHADO ANDREA \\
\hline JAIRON NASCIMENTO ALENCAR & JULIO DE PAIVAMAIA \\
\hline JAMIL ABDALLA SAAD & JUNIOR CAMILO DE QUEIROZ \\
\hline JAMIL RIBEIRO CADE & KLEBER BOMFIM ARAUJO MARTINS \\
\hline JOAO ADDISON PESSOA & LA HORE CORREA RODRIGUES \\
\hline JOAO ALEXANDRE FARJALLA CARACAS & LAERCIO FONSECA ANTELO \\
\hline JOAO ALFREDO FALCAO CUNHA LIMA & LAZARO CLAUDOVINO GARCIA \\
\hline JOAO BATISTA DE OLIVEIRA NETO & LEANDRO ASSUNPCAO CORTES \\
\hline JOAO BATISTA FREITAS GUIMARÃES & LEANDRO COUMBIS MANDALOUFAS \\
\hline JOAO BATISTA LOPES LOURES & LEANDRO DE CARVALHO PEREIRA \\
\hline JOAO BOSCO DA SILVA FILHO & LEANDRO LOBAO LUZ FILHO \\
\hline JOAO CARLOS BELO LISBOA DIAS & LEONARDO ALVES \\
\hline JOAO EDUARDO TINOCO DE PAULA & LEONARDO ALVES BATISTA \\
\hline JOAO FELIPE BARROS DE TOLEDO & LEONARDO AVANY NUNES \\
\hline JOAO FRANCISCO DE SOUZA & LEONARDO COGO BECK \\
\hline JOAO GUILHERME ALVES LOURES & LEONARDO FURTADO OLIVEIRA \\
\hline JOAO LOURENÇO VILLARI HERRMANN & LEONARDO JOSE DUARTE SILVA \\
\hline JOAO LUCAS O CONNELL & LEONARDO MARTINS BARROSO \\
\hline JOAO LUIZ DE ALENCAR ARARIPE FALCAO & LEONIDAS ALVARENGA HENRIQUES \\
\hline JOAO MIGUEL MALTA DANTAS & LIVIA R.FERNANDES \\
\hline JOAO ORAVIO DE FREITAS JUNIOR & LIVIA RIBEIRO FERNANDES \\
\hline JOAO PAULOZOUVI & LUCIANA CONSTANT DAHER \\
\hline JOCELINO PEREGRINO SOARES & LUCIANO DE MOURA SANTOS \\
\hline JORGE DE CAMARGO NETO & LUCIANO MAURICIO DE ABREU FILHO \\
\hline JORGE LUIS HADDAD & LUCIANO NOGUEIRA LIBERATO DE SOUSA \\
\hline JORGE PEREGRINO BRAGA & LUDIMILA PEREIRA TARTUCE \\
\hline JOSE AIRTON DE ARRUDA & LUDMILLA ALMEIDA ROCHA RIBEIRO DE OLIVEIRA \\
\hline JOSE ALBINO MALTA DE SOUZA & LUIS ANTONIO OLIVEIRA FERREIRA \\
\hline JOSE ALFREDO SEJOPOLES & LUIS GUSTAVO DE MIRANDA MARQUES \\
\hline JOSE ANTONIO JATENE & LUIS MARIA CABRERA YORDI \\
\hline JOSE ANTONIO MARIN NETO & LUIZ ALBERTO PIVA E MATTOS \\
\hline JOSE ARMANDO MANGIONE & LUIZ ANTONIO GUBOLINO \\
\hline
\end{tabular}


APÊNDICE (continuação)

Lista de Associados da SBHCI que se mantiveram efetivamente contribuindo com dados à CENIC, durante o biênio 2006-2007

\begin{tabular}{|c|c|}
\hline LUIZ CARLOS TELES CORREA & OSCAR BITTENCOURT LINS NETO \\
\hline LUIZ CLAUDIO MENDES CARVALHO & OTAVIOEBOLI \\
\hline LUIZ EDUARDO KOENIG SAO THIAGO & PABLO TOME TEIXEIRENSE \\
\hline LUIZ EMILIO SALOME & PAULO HENRIQUE JORGE \\
\hline LUIZ HEITOR DEMOLINARI JUNIOR & PAULO MAURICIO PIA DE ANDRADE \\
\hline LUIZ JUNYA KAJITA & PAULO RENATO MERCIO MACHADO \\
\hline LUIZ KOHN & PAULO RICARDO AVANCINI CARAMORI \\
\hline MAEVE DE BARROS CORREIA & PAULO RICARDO FRANCIOZI DE GOIS \\
\hline MANOEL AUGUSTO BAPTISTA ESTEVES & PAULO ROBERTO FERREIRA TARTUCE FILHO \\
\hline MANUEL NICOLAS CANO & PAULO ROGERIO DE OLIVEIRA MONTEIRO \\
\hline MARCEL ROGERS RAVANELLI & PAULO SERGIO DE OLIVEIRA \\
\hline MARCELO BASTOS BRITO & PEDRO ABILIO RIBEIRO RESECK \\
\hline MARCELO EMILIO ARNDT & PEDRO ALVES LEMOS NETO \\
\hline MARCELO GOES ALVES DA SILVA & PEDRO AUGUSTO PASCOLI \\
\hline MARCELO JOSE CANTARELLI & PEDRO BERALDO DE ANDRADE \\
\hline MARCELO LEMOS RIBEIRO & PEDRO EDUARDO HORTA \\
\hline MARCELO SABEDOTTI & PEDRO ESBERARD ARAGAO BELTRAO \\
\hline MARCIO ANDRADE DE OLIVEIRA & PEDRO G./ANA P.LEAL \\
\hline MARCIO ANTONIO DOS SANTOS & PEDRO GOMES DE ALMEIDA GARZON \\
\hline MARCIO AUGUSTO TRUFFA & PEDRO PAULO NEVES DE CASTRO \\
\hline MARCIO JOSE MONTENEGRO DA COSTA & RAIMUNDO ANTONIO DE MELO \\
\hline MARCO ANTONIO NAZARE CASTRO & RALDIR BASTOS FILHO \\
\hline MARCO ANTONIO PERIN & REGIS ARY MOSSMANN \\
\hline MARCO CESAR MIGUITA & RENATO GIESTAS SERPA \\
\hline MARCOS ANTONIO MARINO & RICARDO CESAR CAVALCANTI \\
\hline MARCOS FLAVIO MOELLMAN RIBEIRO & RICARDO JOSE ARAUJO SILVEIRA \\
\hline $\begin{array}{l}\text { MARCOS FRANCHETTI } \\
\text { MARCOS }\end{array}$ & RICARDO JOSE TOFANO \\
\hline MARCOS KYOSHI SUMITA & RICARDO LASEVITCH \\
\hline MARCUS NOGUEIRA DA GAMA & RICARDO MONTEIRO LOURENCO \\
\hline MARDEN ANDRE TEBET & RICARDO PERESSONI FARACO \\
\hline MARIA CRISTINA MEIRA FERREIRA & RICARDO UEDA \\
\hline MARIA DA CONCEICAO ALVES PINTO & RICARDO ZAUITH SILVA \\
\hline MARIA SANALI MOURA DE OLIVEIRA PAIVA & RIZZIERI MOURA GOMES \\
\hline MARIOI.M.BONILLA & ROBERTO DE ALMEIDA CESAR \\
\hline MATEUS ROSSATO & ROBERTO JOSE DA PAIXAO \\
\hline MAURICIO LOPES PRUDENTE & ROBERTO JOSE DE QUEIROZ CREPALDI \\
\hline MAURICIO REZENDE BARBOSA & ROBERTO LEO DA SILVA \\
\hline MAURO ISOLANI PENA & ROBERTO LUCIO DE GUSMAO VERCOZA \\
\hline MICHELI ZANOTI GALON & ROBERTO LUCIO DE GUSMÃO VERÇOZA \\
\hline MICHELI ZANOTTI GALON & ROBERTO OTSUBO \\
\hline MIGUEL ANTONIO NEVES RATI & ROBERTO REIS VIEIRA \\
\hline MILTON DE MACEDO SOARES NETO & ROBERTO VIEIRA BOTELHO \\
\hline MILTON FERREIRA NEVES FILHO & ROBSON BUENO DE CARVALHO \\
\hline MONICA BUCHALLA & RODOLFO ALBERTO SILVEIRA MALTA ALENCAR \\
\hline MOYSES DE OLIVEIRA LIMA FILHO & RODOLFO DE FRANCO CARDOSO \\
\hline MURILLO KENJI FURUKAWA & RODOLFOSTAICO \\
\hline NAHALIEL PESSOA RODRIGUES & RODRIGO COSTA QUINTAO \\
\hline NILSON BORGES RAMOS & RODRIGO DE FRANCO CARDOSO \\
\hline NILSON DE MOURA FE FILHO & RODRIGO JULIO CERCI \\
\hline NILTON CARLOS SPINOLA MACHADO & RODRIGO TRAJANO SANDOVAL PEIXOTO \\
\hline NORBERTO TOAZZA DUDA & RODRIGO VERNEY CASTELLO BRANCO \\
\hline NORIAKITAKESHITA & ROGERIO DE BARROS WANDERLEY \\
\hline
\end{tabular}


APÊNDICE (continuação)

Lista de Associados da $\mathrm{SBHCl}$ que se mantiveram efetivamente contribuindo com dados à CENIC, durante o biênio 2006-2007

ROGERIO DE CASTRO PIMENTEL

ROGERIO EDUARDO GOMES SARMENTO LEITE

ROGERIO FELIPPE TIOSSI

RONE MARQUES PADILHA

RONEI BOSCO DE MATOS

ROSALY GONÇALVES

SALVADOR ANDRE BAVARESCO CRISTOVAO

SAMIR SEME ARAB REIS

SANDRO ANTONIO TEIXEIRA

SANDRO OLIVEIRA SACRE

SERGIO CORREA PRATA

SERGIO GUSTAVO TARBINE

SERGIO KREIMER

SERGIO LUIS BERTI

SIDNEY MUNHOZ JUNIOR

SILVIA MACHADO ABREU

SILVIO GIOPATTO

SILVIO SERGIO PONTES CAMARA

STEFAN COSTA DA SILVEIRA

TAMMUZFATTAH

TARCIO FIGUEIREDO SILVA

TARCISIO MARIZ MAIA FILHO
THIAGO NOBREGA DE OLIVEIRA

TIAGO PORTO DI NUCCI

TRAJANO ALFONSO

UBIRAJARA LIMA FILHO

ULISES ENRIQUE ACUNA SOLORZANO

VAGNER VINICIUS FERREIRA

VALDEMAR DE SOUZA OLIVEIRA JUNIOR

VALERIO FUKS

VALTER CORREIA DE LIMA

VICTOR DUTRA VIEIRA FILHO

VINICIUS DAHER VAZ

VINICIUS FRAGA MAURO

VIRGILIO RIBEIRO FRANCO JUNIOR

VITOR GOMES BARRETO

VITOR OSORIO GOMES

WALASSE ROCHA VIEIRA

WALKIMAR URURAY GLORIA VELOSO

WALTER BENEDUZZI FIOROTTO

WELLINGTON BORGES CUSTODIO

WENCESLAU DE CARVALHO RIBAS

WESLEY FERRAZ DA SILVEIRA

WILSON ALBINO PIMENTEL FILHO 